\section{PROGRAMAS DE APRENDIZAGEM \\ PROFISSIONAL PARA JOVENS: \\ PROPOSTA DE MAPEAMENTO DA PRODUÇÃO CIENTÍFICA SOBRE O TEMA}

PROFESSIONAL APPRENTICESHIP PROGRAMS FOR YOUNG PEOPLE: PROPOSAL FOR MAPPING THE SCIENTIFIC PRODUCTION ABOUT THE

\begin{abstract}
Resumo
O presente trabalho tem por objetivo caracterizar a produção científica referente aos programas de aprendizagem profissional para jovens por meio da análise de artigos disponíveis em bases de dados online. Metodologicamente a pesquisa se enquadra como de natureza exploratória, adotando-se a revisão bibliográfica dos artigos publicados nas bases de dados Science Direct, Scopus, Web of Science e Google Scholar entre os anos de 2006 e 2016. Para a realização dos estudos de produção, autoria, conteúdo e referências dos artigos selecionados foi empregada a técnica de análise bibliométrica. Como resultado, constatou-se a quantidade restrita de artigos publicados sobre programas de aprendizagem profissional para jovens, bem como a existência de lacunas de pesquisa.
\end{abstract}

Palavras-chave: Programa de aprendizagem. Jovens. bibliometria.

\begin{abstract}
This research describes the scientific production about apprenticeship programs to young people by analysis of articles available on databases online. About the methodology, the research is classified as exploratory, adopting the bibliographic review of the articles published in the databases Science Direct, Scopus, Web of Science and Google Scholar in the period of 2006 until the year 2016. To carry out the examination of the production, of the authors, the content and the references of selected article it was proceed a bibliometric analysis. As result, it was found the restrict quantity of articles published about apprenticeship programs to young persons, as well the slack of studies about the issue and opportunities to new studies on the subject.
\end{abstract}

Key-words: Apprenticeship program. Youngers. bibliometrics.

\footnotetext{
${ }^{1}$ Professor Associado de Ciências da Gestão na Universidade Federal do Rio Grande (FURG) e chefe de seu grupo de pesquisa em Pesquisa Operacional (LabSADi). E-mail: longaray@yahoo.com.br

${ }^{2}$ Bacharel em Administração pela Universidade Federal do Rio Grande - FURG. E-mail: belleza.nataly@gmail.com

${ }^{3}$ Professor adjunto da Universidade Federal do Rio Grande. Especialista em Tecnologia Educacional E-mail: paulorsmunhoz@gmail.com

${ }^{4}$ Doutor em administração pela Unisinos. E-mail: vtondolo@gmail.com

${ }^{5}$ Universidade Federal do Rio Grande - FURG, Bolsista de Iniciação Científica da FAPERGS. E-mail: thauaneadamoli@gmail.com
}

Artigo recebido em: 04 de março de 2018. Artigo aceito em 30 de novembro de 2018. 


\section{Introdução}

Devido à competitividade existente no mercado de trabalho, a escolha de profissionais está mais exigente. $\mathrm{O}$ cenário econômico atual impõe às organizações a necessidade de profissionais qualificados, pois em um ambiente competitivo o uso da força de trabalho qualificada se constitui em uma ação estratégica para obtenção de vantagem competitiva. Desta forma, a qualificação profissional não é um fator que contribui apenas com o aprimoramento organizacional, mas também com o desenvolvimento econômico.

No Brasil a falta de qualificação e de experiência profissional, acompanhada da pouca idade, dificulta a inserção do jovem no mercado de trabalho. Dados da Pesquisa Nacional por Amostra de Domicílios Contínua (PNAD, 2018), demonstram que, no segundo trimestre de 2018, jovens entre 14 e 17 anos representaram 8,1\% das pessoas que não possuem atividades de trabalho remunerada. Pessoas entre 18 e 24 anos compõem 32\% desta população. Estes índices de jovens sem ocupação de trabalho vêm aumentando desde o ano de 2012 - contudo, ao comparar o segundo trimestre de 2016 com o segundo trimestre de 2018, houve uma redução de 1,4\% de mão de obra entre 14 e 17 anos sem ocupação de trabalho.

$\mathrm{Na}$ tentativa de reduzir tais índices e propiciar a preparação e inserção ao mercado de trabalho para o jovem foi sancionada a Lei Federal $n^{\circ} 10.097$ (2000), chamada "Lei da Aprendizagem". A legislação determina que empresas de médio e grande porte contratem jovens de 14 a 24 anos para sua capacitação profissional prática e teórica, cumprindo cotas que variam de $5 \%$ a $15 \%$ do número total de funcionários efetivos qualificados, de acordo com os dados do Portal do Ministério do Trabalho e Emprego (MTE).

A partir da Lei da Aprendizagem foi criado o Programa Jovem Aprendiz, que conta com os Sistemas Nacionais de Aprendizagem, entidades qualificadas de formação técnico-profissional e entidades sem fins lucrativos, que instrui os jovens a se inserirem no mercado de trabalho e contribui com sua qualificação profissional. Em dados do documento do Boletim da Aprendizagem Profissional, do MTE (2017), na modalidade de aprendizes contratados do ano de 2005 até o mês de setembro de 2017 houve 3.159.497 contratações de aprendizes no Brasil.

Segundo dados do MTE, no primeiro semestre de 2015 foi superada a meta estabelecida do Plano Plurianual 2012-2015, de inserir no mercado de trabalho 
1.220.628 jovens. Agora o novo intento é de inserir até 2019 o contingente de 1.700 .000 aprendizes, ampliando ainda mais as oportunidades de qualificação profissional e de acesso ao mercado de trabalho para jovens e adolescentes.

De acordo com dados estatísticos do MTE, mais de 50\% dos aprendizes são efetivados nas empresas após o término do seu contrato e $84 \%$ dos jovens continuam no mercado de trabalho, com contrato formal. Esses dados mostram que ainda que, vinculado a uma obrigatoriedade legal, o programa vem contribuindo com a inserção e continuidade dos jovens no mercado de trabalho.

Estudos já realizados sobre o tema no Brasil mostram priorização nas questões sociais e nas demandas sobre as responsabilidades corporativas diante desta política pública. Dentro dessa perspectiva, tem-se a seguinte tese de pesquisa: quais as características da produção científica internacional acerca da temática de Programas de Aprendizagem Profissional para Jovens?

Em face desse panorama, o presente estudo tem por objetivo geral caracterizar a produção cientifica referente aos Programas de Aprendizagem Profissional para Jovens no período entre 2006 e 2016. Os objetivos específicos são: identificar qual a relevância no cenário internacional, a partir da busca de publicações nas bases de dados: Scopus, Web of Science, Science Direct e Google Scholar, sobre programas de aprendizagem profissional para jovens de modo a analisar a partir da análise bibliométrica quais os países, universidades, periódicos e autores mais relevantes dentro da temática. Ao realizar o mapeamento da produção cientifica internacional tem-se a finalidade de mensurar de que forma os programas de aprendizagem profissional estão sendo abordados em outros países.

O artigo está dividido em cinco seções, iniciando com a introdução na seção 1 . A parte 2 apresenta o referencial teórico referente aos Programas de Aprendizagem Profissional para Jovens. A seção 3 realiza o enquadramento metodológico da pesquisa, tendo como base ao detalhamento a análise bibliométrica. A parte 4 descreve os procedimentos metodológicos da pesquisa. E por fim, a quinta apresenta as considerações finais do trabalho, assim como as limitações encontradas e sugestões para futuros estudos.

\section{REFERENCIAL TEÓRICO}

O mercado de trabalho tem exigido, nos últimos anos, um novo perfil do 
trabalhador, que deve ser mais participativo, flexível, diversificado, tecnológico, competitivo, barato, disponível e escolarizado, descrevendo um novo contexto que tende a cobrar mais do trabalhador a partir de uma remuneração mais baixa (DE MOURA, 2018).

Desse modo, a inserção no mercado de trabalho atual destaca exigências referentes à qualificação profissional. Em tempos de crises socioeconômicas a falta de emprego dificulta o ingresso no mercado de trabalho para todas as pessoas com idade produtiva. Porém, para os jovens este cenário ainda é um pouco mais agravante. Segundo Reis (2015), os jovens que não possuem experiência profissional têm probabilidades mais baixas de saírem do desemprego se comparados a indivíduos na mesma faixa etária que já possuem alguma experiência de trabalho. Essa dificuldade em busca do primeiro emprego é ainda maior para os trabalhos nos setores formais, com contrato por tempo indeterminado e em tempo integral.

Na perspectiva de Askilden e Nilsen (2005), o desemprego para os jovens é um problema existente em vários países e suas consequências são mais severas durante períodos de crises. Os autores afirmam também que a razão para o problema do desemprego pode ser encontrada na falta de habilidades dos jovens e na formação de seus salários, que são mais elevados do que a produtividade dos moços contratados.

Com o objetivo de reduzir os índices crescentes de desemprego na juventude, governos de diversos países passaram a prestar mais atenção a programas de formação profissional. Nos Estados Unidos, Barack Obama propôs um "Community College to Career Fund" de US \$ 8 bilhões para promover o ensino profissional. O governo britânico patrocinou uma nova revisão de aprendizagem. Na Coréia do Sul, uma nova rede de escolas profissionais foi estabelecida (BACKES-GELLNER, 2014).

No Brasil também não foi diferente. Políticas nacionais passaram a repensar sobre o desenvolvimento pessoal do jovem que, sem uma profissionalização mediada pela formação educacional técnica formalizada, poderia se tornar socialmente desfavorecido. Desta forma, os benefícios provenientes do programa "Jovem Aprendiz" - que constitui diversificadas possibilidades de inserção no mercado de trabalho contribui por resultar no desenvolvimento profissional dos jovens, mesmo que não garanta a efetivação após o término da sua participação no programa (MIRANDA, 2015).

Nos países da Europa, a aprendizagem também desempenha um papel 
significativo no desenvolvimento de competências e formação de jovens. Os países mais importantes como a Áustria, a Alemanha e a Suíça, são os que contam com o sistema dual de aprendizagem, que se refere ao fato de os aprendizes serem formados e educados em dois locais simultaneamente, no local de trabalho e em escolas profissionais. A França, a Dinamarca, os Países Baixos e o Reino Unido também têm programas de aprendizagem e contam com um número significativo de aprendizes (STEEDMAN, 2005).

Em cada país os modelos de aprendizagem são instruídos de formas diferentes. $\mathrm{Na}$ Europa os programas de aprendizagem combinam com a educação nas instituições educadoras e no local de trabalho e uma parte dos modelos de aprendizagem é predominantemente escolar e apenas apresenta estágios de trabalho curtos. Também há outros modelos que exigem que os aprendizes gastem até $80 \%$ do seu tempo com a empresa de formação (MUEHLEMANN; WOLTER, 2014).

De acordo com Steedman, Gospel e Ryan (1998), a conclusão bem sucedida do programa de aprendizagem leva ao reconhecimento das competências adquiridas por meio de processos de certificação em nível nacional. $\mathrm{O}$ aprendizado eficiente tem a capacidade de desenvolver potencialidades e aspirações que muitas vezes são negligenciadas ou insuficientes apenas com a educação escolar.

Percebe-se que os programas de aprendizagem contribuem significativamente com o desenvolvimento dos jovens ingressantes no mercado de trabalho e contam com o investimento das empresas participantes no processo de formação. Sendo assim, sob a ótica de custo-benefício em relação aos investimentos das empresas na aprendizagem, Muehlemann e Wolter (2014) afirmam que, em vários países europeus, as despesas relacionadas com a formação em aprendizagem são suportadas inteiramente pelo governo ou as empresas têm o reembolso de suas despesas durante o período de formação.

Ainda sob a mesma ótica, Muehlemann e Wolter (2014) esclarecem que em outros países, especialmente quando os aprendizes passam mais tempo em formação, no setor privado realiza-se investimentos substanciais em treinamento de aprendizes, mas espera-se retornos correspondentes em seus investimentos.

Na Suíça, estudos recentes sobre o sistema de aprendizagem nacional mostram que cerca de dois terços das empresas de formação consideram rentável treinar aprendizes (SCHWERI et al., 2003). Isto sugere que para a maioria das empresas de 
LONGARAY; LOURENÇO; MUNHOZ; TONDOLO; AMARAL

formação a possibilidade de recuperar o investimento durante o período de aprendizagem é um fator importante que explica a sua disponibilidade para a formação. Oportuno ressaltar que nem todas as empresas são capazes de oferecer postos de aprendizagem nas mesmas condições favoráveis (MUEHLEMANN et al., 2007).

Já Askilden e Nilsen (2005) acreditam que as empresas treinam os aprendizes com base em decisões de investimento a longo prazo, esperando que o recrutamento de aprendizes seja constante ao longo do ciclo econômico. No entanto, os dados indicam o contrário. Os autores reforçam que as empresas não estariam considerando o recrutamento de aprendizes como um investimento a longo prazo, mas sim como cumprimento de contrato, uma vez que os aprendizes podem também ser utilizados para fins produtivos e quando a formação de aprendizes é subsidiada, o recrutamento de aprendizes pode ser uma forma de as empresas obterem mão-de-obra a custo relativamente mais baixo.

\section{METODOLOGIA}

Com base na estrutura proposta por Gil (2010), os procedimentos metodológicos deste artigo podem ser descritos segundo a sua finalidade, seu objetivo e os métodos adotados. Quanto à finalidade, a pesquisa é classificada como básica, que, segundo Gil (2010), se destina apenas à ampliação do conhecimento, sem preocupação com possíveis benefícios. Assim alinha-se com o objetivo deste estudo, de caracterizar produção cientifica referente aos Programas de Aprendizagem Profissional para Jovens, no período entre 2006 e 2016.

Esta averiguação é classificada como quantitativa, pois as informações dos artigos selecionados são convertidas em dados estatísticos para serem analisados. O delineamento adotado para essa pesquisa é o exploratório, e na visão de Gil (2010) "as pesquisas exploratórias têm como proposito proporcionar maior familiaridade com o problema, com vistas a torná-lo mais explícito ou a construir hipóteses”. A população desta observação é composta pelos artigos selecionados para a realização da análise bibliométrica.

Para a coleta de dados foi utilizada a pesquisa bibliográfica, caracterizando-se como a fonte secundária de dados da pesquisa. Esta técnica mostrou-se a mais adequada, pois no entendimento de Gil (2010), “a pesquisa bibliográfica é desenvolvida com base em material já publicado. Tradicionalmente, esta modalidade de pesquisa 
LONGARAY; LOURENÇO; MUNHOZ; TONDOLO; AMARAL

inclui material impresso, como livros, revistas, jornais, teses, dissertações e anais de eventos científicos". Os dados foram analisados por meio da técnica de análise bibliométrica, em que as informações necessárias foram extraídas para a elaboração desta pesquisa.

\section{ANÁLISE BIBLIOMÉTRICA}

A realização dessa exploração incide na concretização de duas fases da análise bibliométrica. A primeira fase destina-se à coleta dos dados necessários ao cumprimento do objetivo da pesquisa. A segunda etapa é composta por apresentar os resultados atingidos por meio dessa análise (KLEINBURG, 2010; LONGARAY et al., 2015).

\subsection{Coleta de Dados}

A coleta de dados desse estudo se baseou na busca de artigos disponibilizados na internet e a procura foi realizada nas bases de dados: Scopus, Web of Science, Science Direct e Google Scholar. A partir de tais bases de dados concretizou-se o levantamento das produções cientificas relacionadas aos programas de aprendizagem mundial para inserção do jovem no mercado de trabalho, no período entre 2006 e 2016. Foram efetivadas quatro pesquisas nas bases de dados Scopus, Web of Science e Science Direct, com as seguintes palavras-chave em inglês para obter maior abrangência: Young Apprentice and Apprenticeship; Work Market and Apprenticeship; Job Training and Apprenticeship; Young Workers and Apprenticeship.

A Tabela 1 apresenta a quantidade de pesquisas feitas, os termos e os resultados obtidos na base de dados Scopus.

Tabela 1 - Pesquisas realizadas na base de dados Scopus

\begin{tabular}{c|cc}
\hline Pesquisa & Termos & Resultado \\
\hline 1 & YOUNG APPRENTICE and APPRENTICESHIP & 50 \\
2 & WORK MARKET and APPRENTICESHIP & 48 \\
3 & JOB TRAINING and APPRENTICESHIP & 80 \\
4 & YOUNG WORKERS and APPRENTICESHIP & 25 \\
\hline
\end{tabular}

Fonte: Dados da pesquisa

Na Tabela 2 apresenta-se a quantidade de pesquisas feitas, os termos e os resultados obtidos na base de dados Web of Science.

Tabela 2 - Pesquisas realizadas na base de dados Web of Science

\begin{tabular}{ccc}
\hline Pesquisa & Termos & Resultado \\
\hline
\end{tabular}

Revista Eletrônica Gestão e Serviços v.9, n. 2, pp. 2512-2532, julho/dezembro 2018.

ISSN Online: 2177-7284 e-mail: regs@ metodista.br 
LONGARAY; LOURENÇO; MUNHOZ; TONDOLO; AMARAL

\begin{tabular}{c|c|c|}
\hline 1 & YOUNG APPRENTICE and APPRENTICESHIP & 22 \\
\hline 2 & WORK MARKET and APPRENTICESHIP & 31 \\
\hline 3 & JOB TRAINING and APPRENTICESHIP & 36 \\
\hline 4 & YOUNG WORKERS and APPRENTICESHIP & 17 \\
\hline
\end{tabular}

Fonte: Dados da pesquisa

A Tabela 3 destaca a quantidade de pesquisas feitas, os termos e os resultados obtidos na base de dados Science Direct.

Tabela 3 - Pesquisas realizadas na base de dados Science Direct

\begin{tabular}{c|cc}
\hline Pesquisa & Termos & Resultado \\
\hline 1 & YOUNG APPRENTICE and APPRENTICESHIP & 4 \\
2 & WORK MARKET and APPRENTICESHIP & 7 \\
3 & JOB TRAINING and APPRENTICESHIP & 7 \\
\hline 4 & YOUNG WORKERS and APPRENTICESHIP & 3 \\
\hline
\end{tabular}

Fonte: Dados da pesquisa

(Este parágrafo contém muitas informações e acabou ficando meio confuso. O problema pode estar ainda na pontuação. Não consegui entender muito bem as informações/os indicadores utilizados, mas tentei melhorá-lo. Gentileza verificar se ficou correto. Se não ficou, sugiro reconstruí-lo.) Para a pesquisa na base de dados Scopus foram utilizados os indicadores: título, resumo e palavras-chave (article, title, abstract, keyboard) e área de ciências sociais e humanidades (social sciences \& humanities). Já na base de dados Web of Science empregou-se os tópicos (topic); na área de ciências sociais (social sciences citation index) e na base de dados Science Direct os indicadores usados foram os resumos, títulos e palavras-chave (abstract, title, keyword) dentro da área de ciências sociais (social science), e Negócios, Gestão e Contabilidade (business, management and accounting).

Realizou-se três pesquisas na base de dados Google Scholar. A procura de artigos foi feita pela busca das seguintes expressões em pares: Young Apprentice and Apprenticeship System; Apprenticeship and Young Workers; Vocational Training and Apprenticeship. O período pesquisado igualmente está entre os anos 2006 e 2016. Na Tabela 4 apresentamos a quantidade de pesquisas, os termos e os resultados obtidos.

Tabela 4 - Pesquisas realizadas na base de dados Google Scholar

\begin{tabular}{c|cc}
\hline Pesquisa & Termos & Resultado \\
\hline 1 & YOUNG APPRENTICE and APPRENTICESHIP & 16.800 \\
2 & APPRENTICESHIP and Young Workers & 25.400 \\
3 & VOCATIONAL TRAINING and APPRENTICESHIP & 16.700 \\
\hline
\end{tabular}

Revista Eletrônica Gestão e Serviços v.9, n. 2, pp. 2512-2532, julho/dezembro 2018. ISSN Online: 2177-7284 e-mail: regs@metodista.br 
Fonte: Dados da pesquisa

Após realizar a pesquisa no Google Scholar os resultados obtidos foram verificados, buscando apenas a existência de artigos científicos que possuíssem alinhamento com a proposta da pesquisa. Monografias, dissertações e teses foram descartadas, assim como trabalhos de anais de congressos e simpósios. Artigos que não se enquadram ao período determinado e de outras áreas de estudo também foram retirados. Por fim, resultou um total de 19 artigos a serem utilizados para este estudo.

As investigações nas bases de dados Scopus, Web of Science e Science Direct, encontraram 330 artigos, sendo que 77 se repetiam e 216 trabalhos não estavam alinhados com o tema da pesquisa ou pertenciam a outras áreas, e foram descartados. Dessas bases de dados foram aproveitados 18 textos. Finalizando o processo de coleta, utilizou-se os artigos das quatro bases de dados (Scopus, Web of Science, Science Direct e Google Scholar), resultando em 37 trabalhos para a análise bibliométrica (Apêndice A).

\subsection{Resultados}

Para analisar detalhadamente os resultados obtidos na pesquisa os dados foram separados em categorias de acordo com o fim do estudo. Assim, eles foram divididos da seguinte maneira: Estudo da produção e autoria; Estudo do conteúdo e, por último, Estudo das referências bibliográficas.

\subsubsection{Estudo da produção e autoria}

Nesta fase do estudo o objetivo foi mensurar o número de publicações por ano e classificar os artigos de acordo com a quantidade de autores por texto. Buscou-se identificar ainda os pesquisadores que mais contribuíram com a produtividade do assunto, relacionando-os de acordo com o número de publicações no período estudado.

Com o objetivo de avaliar a produção científica com relação aos programas de aprendizagem mundial para jovens no mercado de trabalho, construiu-se um histograma de acordo com o número de publicações identificadas por ano. Adotou-se a curva de melhor ajuste, usando como critério o maior valor de $\mathrm{R}^{2}$. Nesse escopo o valor de $\mathrm{R}^{2}$ aponta a porcentagem da modificação da variável (Número de artigos) que é explicada pela variável independente (ano), como pode ser observado na Figura 1. 


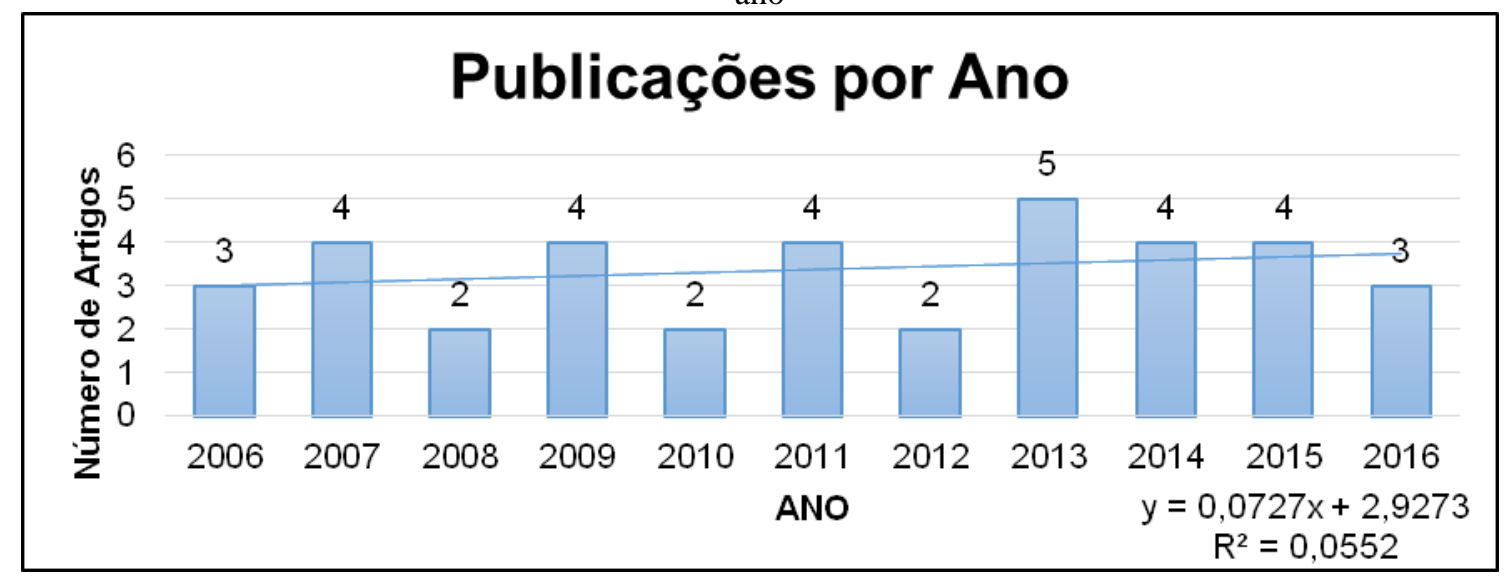

Fonte: Dados da pesquisa

Verificou-se que a maioria dos artigos foi produzida por um ou dois autores $(69,99 \%)$ e a menor parte deles, escrita por quatro ou cinco pesquisadores $(11,85 \%)$, conforme demonstrado na Tabela 5.

Tabela 5 - Número de autores por artigo

\begin{tabular}{ccc}
\hline Número de Autores & Artigos & $\%$ \\
\hline 1 & 11 & 29,7 \\
2 & 11 & 29,7 \\
3 & 9 & 24,3 \\
4 & 4 & 10,8 \\
\hline 5 & 2 & 5,4 \\
\hline Total & 37 & 100 \\
\hline
\end{tabular}

Fonte: Dados da pesquisa

Com o objetivo de identificar os autores que mais contribuíram com a produção de artigos do assunto estudado relacionou-se os estudiosos com mais participações na amostra de trabalhos. A Tabela 6 relaciona os nomes dos autores que apareceram no mínimo duas vezes e suas porcentagens no âmbito total.

Destacam-se como autores mais prolíferos: Wolter, S. C, com 6 participações; Muehlemann, S. (com 5); Schvveri, J. (com 3); Chan, S., Kammermann, M., Mohrenweiser, J., Winch, C (com 2).

Tabela 6 - Autores com mais participações na amostra

\begin{tabular}{ccc}
\hline Autores & N & $\%$ \\
\hline Wolter, S. C. & 6 & $6,98 \%$ \\
\hline Muehlemann, S. & 5 & $5,81 \%$ \\
\hline Schvveri, J. & 3 & $3,49 \%$ \\
\hline Chan, S. & 2 & $2,33 \%$ \\
\hline
\end{tabular}


LONGARAY; LOURENÇO; MUNHOZ; TONDOLO; AMARAL

\begin{tabular}{|c|c|c|}
\hline Kammermann, M. & 2 & $2,33 \%$ \\
\hline Mohrenweiser, J. & 2 & $2,33 \%$ \\
\hline Winch, C. & 2 & $2,33 \%$ \\
\hline Total & 22 & $25,60 \%$ \\
\hline
\end{tabular}

Fonte: Dados da pesquisa

Analisando a produção acadêmica por instituições, verificou-se as universidades que mais contribuíram com pesquisas referentes ao presente estudo, a partir das instituições em que os autores dos artigos encontravam-se associados. Foram identificadas 47 e as que apresentaram maior participação são: University of Bern, com 14,89\% de participação; Swiss Federal Institute for Vocational Education and Training, com 6,38\% de participação; Akdeniz University, Christchurch Polytechnic Institute of Technology, Universität Zürich e University of Bonn, com 4,26\% respectivamente. Demonstra-se na Tabela 7 o percentual e o número de vezes em que as instituições aparecem.

Tabela 7 - Universidades com mais participação na amostra

\begin{tabular}{|c|c|c|}
\hline Instituições & Total & $\%$ \\
\hline University of Bern & 7 & 14,89 \\
\hline Swiss Federal Institute for Vocational Education and Training & 3 & 6,38 \\
\hline Akdeniz University & 2 & 4,26 \\
\hline Christchurch Polytechnic Institute of Technology & 2 & 4,26 \\
\hline Universität Zürich & 2 & 4,26 \\
\hline University of Bonn & 2 & 4,26 \\
\hline
\end{tabular}

Fonte: Dados da pesquisa

\subsubsection{Estudo do conteúdo}

Nesta etapa do estudo analisou-se o conteúdo dos artigos com a finalidade de verificar quais são os temas mais recorrentes no período de 2006 e 2016. As palavraschave que obtiveram maior frequência nos artigos selecionados e as metodologias mais utilizadas nas pesquisas igualmente foram verificadas.

O tema mais frequente, com um total de 8 artigos, foi a Análise Econômica dos Programas de Aprendizagem, com percentual de participação de 22,22\%. Seguido dos temas Impactos dos Programas de Aprendizagem, com 5 artigos e 13,89\% de participação; Processo de inserção no Trabalho, com 4 artigos e 11,11\% de participação; e Analise Comportamental, Desenvolvimento do Capital Humano e Qualificação profissional, com 2 artigos cada um e percentual de participação de 5,56\% (Tabela 8). 
Tabela 8 - Resumo dos temas abordados nos artigos selecionados

\begin{tabular}{|c|c|c|}
\hline Temas & Total & $\%$ \\
\hline Análise Econômica dos Programas de Aprendizagem & 8 & 22,22 \\
\hline Impactos dos Programas de Aprendizagem & 5 & 13,89 \\
\hline Processo de inserção no Trabalho & 4 & 11,11 \\
\hline Análise Comportamental & 2 & 5,56 \\
\hline Desenvolvimento do Capital Humano & 2 & 5,56 \\
\hline Estratégias na Formação/Treinamento & 2 & 5,56 \\
\hline Qualificação profissional & 2 & 5,56 \\
\hline Total & 25 & 69,44 \\
\hline
\end{tabular}

Fonte: Dados da pesquisa

Após o estudo dos temas mais recorrentes nos artigos da amostra analisaram-se as palavras-chave mais frequentes nos textos - resultaram selecionadas as palavraschave que possuíam no mínimo 2 artigos, conforme demonstrado na Tabela 9.

A palavra-chave que apareceu com maior frequência foi Apprenticeship training, com um total de 10 vezes (percentual de representação de 11,11\%). Em seguida, Apprenticeship, com um total de 8 vezes e (percentual de representação de 8,89\%). As palavras-chave Cost-benefit analysis, Disadvantaged youth e Vocational education and training - VET, com um total de 3 vezes (representam 3,33\%). Por fim, Human capital, Programm e evaluation e Regional labor markets, com um total de duas vezes $(2,22 \%)$. Ao todo foram identificadas 90 palavras-chave.

Tabela 9 - Resumo das palavras-chave identificadas nos artigos

\begin{tabular}{|c|cc|}
\hline Palavras-Chave & Total & $\%$ \\
\hline Apprenticeship training & 10 & 11,11 \\
\hline Apprenticeship & 8 & 8,89 \\
\hline Cost-benefit analysis & 3 & 3,33 \\
\hline Disadvantaged youth & 3 & 3,33 \\
\hline Vocational education and training - VET & 3 & 3,33 \\
\hline Human capital & 2 & 2,22 \\
\hline Programme evaluation & 2 & 2,22 \\
\hline Regional labor markets & 2 & 2,22 \\
\hline School-to-work transition & 2 & 2,22 \\
\hline Vocational education & 2 & 2,22 \\
\hline Total & 37 & 41,11 \\
\hline
\end{tabular}

Fonte: Dados da pesquisa.

\subsubsection{Estudos das Referências bibliográficas}

Revista Eletrônica Gestão e Serviços v.9, n. 2, pp. 2512-2532, julho/dezembro 2018.

ISSN Online: 2177-7284 e-mail: regs@metodista.br 
LONGARAY; LOURENÇO; MUNHOZ; TONDOLO; AMARAL

Na etapa do estudo das referências bibliográficas dos 37 artigos selecionados o objetivo foi identificar as obras de maior relevância na área. Foram analisadas ao total 1.363 referências dos artigos relacionados a pessoas físicas. Na Tabela 10 descreve-se as obras de maior repercussão, a quantidade de vezes em que são citadas, os autores, o título da obra e o tipo da mesma.

Tabela 10 - Obras de maior repercussão entre os artigos selecionados

\begin{tabular}{|c|c|c|c|}
\hline Citações & Autores & Titulo & Tipo \\
\hline 11 & Acemoglu D., Pischke J. s. & Why do firms train? Theory and evidence & Artigo \\
\hline 10 & $\begin{array}{c}\text { Schoenfeld G., Wenzelman F., } \\
\text { Dionisus R., Pfeifer H. and } \\
\text { Walden G. }\end{array}$ & $\begin{array}{l}\text { Kosten und Nutzen der dualen Ausbildung aus } \\
\text { Sicht der Betriebe }\end{array}$ & Livro \\
\hline 8 & Ryan P. & $\begin{array}{l}\text { The School-to-Work Transition: A Cross- } \\
\text { National Perspective }\end{array}$ & Artigo \\
\hline 7 & Ryan P. & $\begin{array}{l}\text { Is Apprenticeship better? A Review of } \\
\text { Economic Evidence }\end{array}$ & Artigo \\
\hline 6 & Beicht, U. et al & $\begin{array}{l}\text { Kosten und Nutzen der betreib- } \\
\text { lichen Berufsausbildung in Deutschland. } \\
\text { Berichte zur beruflichen Bildung }\end{array}$ & Artigo \\
\hline 6 & Kuczera, M. & $\begin{array}{l}\text { Learning for jobs OECD review of vocational } \\
\text { education and training. }\end{array}$ & Artigo \\
\hline 6 & Acemoglu D and Pischke J-S & $\begin{array}{l}\text { The structure of wages and investment in } \\
\text { general training }\end{array}$ & Artigo \\
\hline
\end{tabular}

Von Bardeleben R, Beicht U and
Fehér K

5 Acemoglu D, Pischke JS

\begin{tabular}{|l|l|l|l}
\hline $\mathbf{5}$ & Winkelmann, R. & $\begin{array}{c}\text { Employment Prospects and Skill acquisition of } \\
\text { Apprenticeship - Trained Workers in Germany }\end{array}$ & Artigo \\
\hline $\mathbf{5}$ & Becker, G.S. & $\begin{array}{c}\text { Investment in human capital: a theoretical } \\
\text { analysis }\end{array}$ & Artigo \\
\hline
\end{tabular}

\section{Betriebliche Kosten und Nutzen der Ausbildung: Repräsentative Resultate aus Artigo Industrie, Handel und Handwerk.}
Beyond Becker: Training in imperfect labour markets
Artigo (1)

\begin{tabular}{l|c|c|c} 
& Wolter S. C., Mühlemann S. and & Why Some Firms Train Apprentices and Many & Artigo \\
Schweri J. & Others Do Not
\end{tabular}

Revista Eletrônica Gestão e Serviços v.9, n. 2, pp. 2512-2532, julho/dezembro 2018.

ISSN Online: 2177-7284 e-mail: regs@ metodista.br 
4

Muehlemann S, Schweri J, Winkelmann R, Wolter SC
An empirical analysis of the decision to train apprentices

Artigo

An investment model for the supply of training by employers.

Artigo

Stevens, M.$$
\text { by employers. }
$$

Econometric Analysis of Cross Section and Panel Data

Artigo

Wooldridge J. M.

From School to Productive Work. Britain and Switzerland Compared

Artigo

Mobility after Apprenticeship - Evidence from Register Data

Artigo

Situated learning: Legitimate peripheral participation

Artigo

Lave, J., \& Wenger, E.

Training and union wages.

Artigo

Apprenticeship training and the business cycle Artigo Wüest A

Apprenticeship.

Livro

3

Wolter S.C. and Ryan P.

Apprenticeship: Between Theory and Practice, School and Workplace

Artigo

3

Ryan P.

School and Workplace

Ausbildungsintensitaet und

Ausbildungsbeteiligung von Betrieben:

3 Neubaeumer, R., \& Bellmann, L.
Theoretische Erklaerungen und empirische

Ergebnisse auf der Basis des IAB-

Betriebspanels 1997.

Artigo

Betriebliche Kosten und Nutzen der Industrie, Handel und Handwerk. Ausbildung: Repräsentative Resultate aus

Artigo Fehér K
3 Caliendo M, Künn S and Schmidl $\mathrm{R}$
Fighting Youth Unemployment: The Effects of Active Labor Market Policies
Artigo

General training by firms, apprentice contracts, and public policy

Artigo McCormick B

Becker, G. S.
Human Capital. A Theoretical and Empirical Analysis, with Special Reference to Education. 
3 Wolter, S. C. and J. Schweri.

The Cost and Benefit of Apprenticeship Training: The Swiss Case

Artigo

The economics of private sector training: A survey of the literature

Artigo

3

Leuven E
Muehlemann S, Pfeifer H,

3 Walden G, Wenzelmann F, Wolter SC
The Financing of Apprenticeship Training in the Light of Labor Market Regulations.
Wage Compression and Firm-Sponsored Training in Germany: Empirical Evidence for the Acemoglu-Pischke Model from a ZeroInflated Count Data Model

\section{Considerações finais}

Este estudo teve por objetivo a caracterização da produção cientifica referente 
LONGARAY; LOURENÇO; MUNHOZ; TONDOLO; AMARAL

aos Programas de Aprendizagem Profissional para Jovens, no período entre 2006 e 2016, por meio de uma análise de artigos disponíveis em bases de dados online. Utilizou-se a técnica da bibliometria na realização do estudo da produção, da autoria, do conteúdo e das referências bibliográficas dos artigos selecionados.

A partir dos resultados encontrados na análise bibliométrica constatou-se que a maioria dos artigos analisados foi escrita por um ou dois autores. Os temas de maior relevância abordados na pesquisa são: a Análise Econômica dos Programas de Aprendizagem, Impactos dos Programas de Aprendizagem e o Processo de inserção no Trabalho. Os autores que mais contribuíram com a produção de artigos do assunto estudado são: Wolter, S. C. (University of Bern), Muehlemann, S. (University of Bern) e Schweri, J. (University of Bern). As universidades que se destacaram com a produção cientifica na temática estudada: University of Bern e Swiss Federal Institute for Vocational Education and Training.

Como limitações para esta pesquisa ressalta-se a reduzida quantidade de artigos analisados devido ao período de tempo definido no trabalho. $\mathrm{O}$ volume de textos não ligados à área da Administração reduziu significativamente o número da amostra, impossibilitando assim, análise mais detalhada sobre o assunto na área de interesse. As referências bibliográficas apresentaram maior dificuldade para análise devido à formatação, padronização e os diferentes idiomas utilizados.

Por meio da bibliometria foi possível identificar como lacuna desta pesquisa que existe um reduzido número de investigação que abarca a temática do Jovem Aprendiz no âmbito da área da Administração.

O tema de pesquisa analisado no presente estudo pode ser desenvolvido em pesquisas futuras, abrangendo um número maior de bases de dados, bem como não se restringir a uma área de estudo afim de suprir alguma lacuna de pesquisa.

Outra possibilidade é o estudo dos artigos que compõem as referências bibliográficas dos trabalhos selecionados, demonstrando aqueles de maior relevância. Por fim, ainda como sugestão para pesquisas futuras a partir da revisão de literatura desenvolvida, sugere-se realizar uma análise da aplicação de programas de jovens aprendizes, tendo em vista mensurar a relevância de iniciativas voltadas à inserção de jovens no mercado de trabalho, mas também, elencar os retornos financeiros das empresas que empregam via programas de jovens aprendizes.

Revista Eletrônica Gestão e Serviços v.9, n. 2, pp. 2512-2532, julho/dezembro 2018.

ISSN Online: 2177-7284 e-mail: regs@ metodista.br 


\section{REFERÊNCIAS}

ASKILDEN, J. E.; NILSEN, O. A. Apprentices and Young Workers: A study of the Norwegian Youth Labour Market. Scottish Journal of Political Economy, v. 52, n. 1, p. 1-17. Oxford, Blackwell Publishing. Fevereiro 2005.

BACKES-GELLNER, U. Benefits of Apprenticeship Training and Future Challenges - Empirical Results and Lessons from Switzerland and Germany. Leading House Working Paper: Zurich, nº 9, p. 1-12. February 2014.

BRASIL. Lei da Aprendizagem. Lei no 10.097, de 19 de dezembro de 2000. Brasília/DF, 2000.

Decreto no 5.598/2005. Brasília: Diário oficial da União, 2005.

DE MOURA, L. S. JUVENTUDE E TRABALHO: O perfil do jovem aprendiz da Rede Pró-Aprendiz-GO. 2018. 131f. Tese (Doutorado em Sociologia) - Faculdade de Ciências Sociais, Universidade de Goiás, Goiás, 2018.

GIL, A. C. Como elaborar projetos de pesquisa. São Paulo: Atlas, 2010.

IBGE; Instituto Brasileiro de Geografia e Estatística. Pesquisa Nacional de Amostra de Domicílios. Disponível em: <https://www.ibge.gov.br/estatisticasnovoportal/sociais/rendimento-despesa-e-consumo/9173-pesquisa-nacional-poramostra-de-domicilios-continua-trimestral.html?edicao $=22193 \& \mathrm{t}=$ destaques $>$. Acesso em: 11 nov. 2018.

LONGARAY, A. A.; POPIOLEK JUNIOR, T. L.; MUNHOZ, P. R.; GERI, F. S.; CASTELLI, T. M. Caracterização da produção científica brasileira sobre a aplicação de métodos multicritério de apoio à decisão: uma análise das publicações entre 2004 - 2013. In: anais do XXXV ENEGEP - Encontro Nacional de Engenharia de Produção. Fortaleza, ABEPRO - Associação Brasileira de Engenharia de Produção, 2015

MTE; Ministério do Trabalho e Emprego. Boletim da Aprendizagem Profissional. Disponível em: <https://centbrasil.org/images/blog/boletim-da-aprendizagemprofissional.pdf >. Acesso em: 11 nov. 2018.

MTE; Ministério do Trabalho e Emprego. Inclusão de aprendizes até 2019.

Disponível em: <http://trabalho.gov.br/noticias/1030-mte-pretende-incluir-1-7-milhaode-aprendizes-ate-2019>._Acesso em: 31 ago. 2016.

MIRANDA, I. S. Adolescente Aprendiz: Pensando a inclusão pelo viés do trabalho. Revista Foco, v. 8, n. 2, p. 116-132. Vila Velha, Faculdade Novo Milênio, 2015.

MUEHLEMANN, S.; WOLTER S. C. Return on investment of apprenticeship systems for enterprises: Evidence from cost-benefit analyses. IZA Journal of Labor Policy, n. 3, v. 25, p. 1-22. Bonn, Springer Open, 2014. 
MUEHLEMANN, S.; SCHERI, J.; WINKELMANN, R.; WOLTER, S. C. An Empirical Analysis of the Decision to Train Apprentices. LABOUR: Review of Labour Economics and Industrial Relations, v. 21, n. 3, p. 419-441. Oxford, CEIS, Fondazione Giacomo Brodolini and John Wiley \& Sons Ltd, 2007.

REIS, M. Uma análise da Transição dos Jovens para o Primeiro Emprego no Brasil. Revista Brasileira de Economia, v. 69, n. 1, p. 125-143. Rio de Janeiro, Fundação Getúlio Vargas - EPGE, 2015.

SCHERI, J. et al. Kosten und Nutzen der Lehrlingsausbildung aus der Sicht Schweizer Betriebe. Beiträge zur Bildungsökonomie, Band 2, Chur und Zürich: Rüegger Verlag, 2003.

STEEDMAN, H. Apprenticeship in Europe: 'Fading' or Flourishing? The London School of Economics and Political Science, London, UK, paper $n^{\circ} 10$. Centre for Economic Performance Special Papers. CEP Discussion, 2005.

STEEDMAN, H.; GOSPEL, H.; RYAN, P. Apprenticeship: a strategy for growth. The London School of Economics and Political Science, London, UK. Centre for Economic Performance Special Papers, CEPSP11, 1998. 
Apêndice A

\begin{tabular}{|c|c|}
\hline ID & Artigo \\
\hline 1 & $\begin{array}{l}\text { Akpınar, T. and S. Gün (2016). "Testing the human capital development } \\
\text { model: the case of apprenticeships in Turkey." International Journal of } \\
\text { Training and Development } 20(3): 214-223 .\end{array}$ \\
\hline 2 & $\begin{array}{l}\text { Amazarray, M. R., et al. (2009). "Apprentice versus worker: Adolescents } \\
\text { in apprenticeship process." Psicologia: Teoria e Pesquisa 25(3): 329-338. }\end{array}$ \\
\hline 3 & $\begin{array}{l}\text { Bessey, D. and Backes-Gellner, U. (2007). Premature Apprenticeship } \\
\text { Terminations - An Economic Analysis. Working Paper No. } 2 .\end{array}$ \\
\hline 4 & $\begin{array}{l}\text { Brockmann, M.; Clarke, L.; Méhaut, P. and Winch, C. (2008). } \\
\text { Competence-Based Vocational Education and Training (VET) - the Cases } \\
\text { of England and France in a European Perspective. Vocations and Learning } \\
\text { (2008) } 1: 227-244\end{array}$ \\
\hline 5 & $\begin{array}{l}\text { Brunello, G. (2009). The effect of economic downturns on apprenticeships } \\
\text { and initial workplace training - a review of the evidence. vocational } \\
\text { education and training } 1 \text { (2009) } 2 \text {, S. } 145-171\end{array}$ \\
\hline 6 & $\begin{array}{l}\text { Cahuc, P.; Carcillo, S.; Zimmermann, K. F. (2013). The Employment of } \\
\text { the Low-Skilled Youth in France. IZA Policy Paper No. } 64\end{array}$ \\
\hline 7 & $\begin{array}{c}\text { Chan, S. (2013). "Learning Through Apprenticeship: Belonging to a } \\
\text { Workplace, Becoming and Being." Vocations and Learning 6(3): 367-383. }\end{array}$ \\
\hline 8 & $\begin{array}{l}\text { Chan, S. (2016). "Belonging to a workplace: first-year apprentices' } \\
\text { perspectives on factors determining engagement and continuation through } \\
\text { apprenticeship." International Journal for Educational and Vocational } \\
\text { Guidance 16(1): 9-27. }\end{array}$ \\
\hline 9 & $\begin{array}{l}\text { Courtinat-Camps, A. and F. Fourchard (2011). "Alternating training and } \\
\text { school specialization: Towards new forms of support to wage-earning } \\
\text { apprenticeship at level 5." Psychologie du Travail et des Organisations } \\
\text { 17(3): 233-251. }\end{array}$ \\
\hline 10 & $\begin{array}{c}\text { Deissinger, T. Smith, E. and Pickersgill, R. (2006). Models of full-time } \\
\text { and part-time vocational training for school-leavers - A comparison } \\
\text { between Germany and Australia. International Journal of Training } \\
\text { Research Volume 4, Number 1, 2006. }\end{array}$ \\
\hline 11 & $\begin{array}{l}\text { Dolado, J. J.; Jansen, M.; Felgueroso, F.;Fuentes, A. and Wölfl, A. (2013). } \\
\text { Youth labour market performance in Spain and its determinants - a micro- } \\
\text { level perspective. OECD Economics Departament Working Papers N. } \\
1039 .\end{array}$ \\
\hline 12 & $\begin{array}{c}\text { Eichhorst, W.; Hinte, H. and Ulf,R. (2013). Youth Unemployment in } \\
\text { Europe - What to Do about It. IZA Policy Paper No. } 65\end{array}$ \\
\hline
\end{tabular}

Revista Eletrônica Gestão e Serviços v.9, n. 2, pp. 2512-2532, julho/dezembro 2018. ISSN Online: 2177-7284 e-mail: regs@ metodista.br 
LONGARAY; LOURENÇO; MUNHOZ; TONDOLO; AMARAL

\begin{tabular}{|c|c|}
\hline 13 & $\begin{array}{l}\text { Fersterer, J. Pischke, J. S. and Winter-Ebmer, R. (2008). Returns to } \\
\text { apprenticeship training in Austria - evidence from failed firms. } \\
\text { Scandinavian journal of economics, } 110 \text { (4). pp. 733-753. }\end{array}$ \\
\hline 1 & $\begin{array}{l}\text { Fitzenberger, B., et al. (2015). "Mobility across firms and occupations } \\
\text { among graduates from apprenticeship." Labour Economics 34: 138-151. }\end{array}$ \\
\hline 15 & $\begin{array}{l}\text { Friberg, K. (2014). "Apprenticeship orientation as planned behavior in } \\
\text { educational choices: A path model of antecedent beliefs." Empirical } \\
\text { Research in Vocational Education and Training } 6(1) .\end{array}$ \\
\hline 16 & $\begin{array}{l}\text { Gray, D. and Morgan, M. (2011). Modern apprenticeships - filling the } \\
\text { skills gap. Journal of Vocational Education and Training, Vol. } 50\end{array}$ \\
\hline 17 & $\begin{array}{c}\text { GunbayI,I. and Ozel, M. (2013). The Problems in TVET for } \\
\text { Apprenticeship from the Perspective. International Vocational Education } \\
\text { and Training Association, } 2013 .\end{array}$ \\
\hline 18 & $\begin{array}{l}\text { Horn, D. (2016). "The effectiveness of apprenticeship training: A within- } \\
\text { track comparison of workplace-based and school-based vocational } \\
\text { training in Hungary." Social Science Research 55: 139-154. }\end{array}$ \\
\hline 19 & $\begin{array}{c}\text { Jansen, A., et al. (2015). "Labour market deregulation and apprenticeship } \\
\text { training: A comparison of German and Swiss employers." European } \\
\text { Journal of Industrial Relations } 21(4): 353-368 .\end{array}$ \\
\hline 20 & $\begin{array}{c}\text { Kammermann, M. (2010). Job or further training Impact of the Swiss } \\
\text { Basic Federal Vocational Education and Training (VET) Certificate on the } \\
\text { careers of low achieving young people. Education + Training, Volume } 53 \\
\text { No. } 5 \text {. }\end{array}$ \\
\hline 21 & $\begin{array}{l}\text { Kammermann, M., et al. (2011). "Two-year apprenticeships - a successful } \\
\text { model of training?" Journal of Vocational Education and Training } \mathbf{6 3}(3) \text { : } \\
377-396 .\end{array}$ \\
\hline 22 & 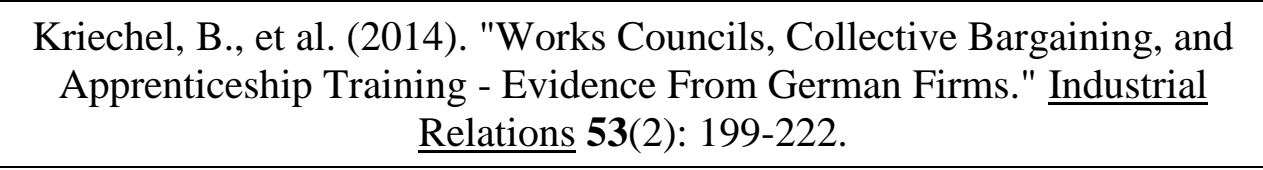 \\
\hline 23 & $\begin{array}{l}\text { Lerman, R. I. (2012). Can the United States Expand Apprenticeship- } \\
\text { Lessons from Experience. IZA Policy Paper No. } 46\end{array}$ \\
\hline 24 & $\begin{array}{l}\text { Mohrenweiser, J. (2012). "Which firms train disadvantaged youth." } \\
\text { Empirical Research in Vocational Education and Training 4(2): 115-130. }\end{array}$ \\
\hline 25 & $\begin{array}{l}\text { Mohrenweiser, J. and F. Pfeiffer (2015). "Coaching disadvantaged young } \\
\text { people: Evidence from firm level data." Jahrbucher fur Nationalokonomie } \\
\text { und Statistik } 235(4-5): 459-473 .\end{array}$ \\
\hline 26 & $\begin{array}{l}\text { Muehlemann, S. and S. C. Wolter (2014). "Return on investment of } \\
\text { apprenticeship systems for enterprises: Evidence from cost-benefit } \\
\text { analyses." IZA Journal of Labor Policy } \mathbf{3}(1) \text {. }\end{array}$ \\
\hline 27 & $\begin{array}{l}\text { Muehlemann, S. and Wolter, S. C. (2006). Regional Effects on Employer } \\
\text { Provided Training - Evidence from Apprenticeship Training In } \\
\text { Switzerland. Cesifo Working Paper N.1665. Category 4: Labour Markets. }\end{array}$ \\
\hline
\end{tabular}

Revista Eletrônica Gestão e Serviços v.9, n. 2, pp. 2512-2532, julho/dezembro 2018. ISSN Online: 2177-7284 e-mail: regs@ metodista.br 
LONGARAY; LOURENÇO; MUNHOZ; TONDOLO; AMARAL

\begin{tabular}{|c|c|}
\hline 28 & $\begin{array}{c}\text { Muehlemann, S.; Schweri, J.; Winkelmann, R. and Wolter, S.C. (2007). } \\
\text { An Empirical Analysis of the Decision to Train Apprentices. Journal } \\
\text { compilation } 21 \text { (3) 419-441 }\end{array}$ \\
\hline 29 & $\begin{array}{l}\text { Muehlemann, S.; Wolter, S. C. and Wüest, A. (2009). Apprenticeship } \\
\text { training and the business cycle. Vocational Education and Training } 2 \\
\text { (2009) 173-186 }\end{array}$ \\
\hline 30 & $\begin{array}{c}\text { Parey, M. (2009). Vocational Schooling versus Apprenticeship Training } \\
\text { — Evidence from Vacancy Data. Journal of Vocational Education and } \\
\text { Training. }\end{array}$ \\
\hline 31 & $\begin{array}{l}\text { Polidano, C. and D. Tabasso (2014). "Making it real: The benefits of } \\
\text { workplace learning in upper-secondary vocational education and training } \\
\text { courses." Economics of Education Review 42: 130-146. }\end{array}$ \\
\hline 32 & $\begin{array}{l}\text { Ridzwan, C. R. and R. M. Yasin (2015). "Cultivating Learning: A } \\
\text { Grounded Theory of Skills Acquisition for Vocation in Modern } \\
\text { Apprenticeships." Procedia - Social and Behavioral Sciences 174: 275- } \\
282 .\end{array}$ \\
\hline 33 & $\begin{array}{l}\text { Schweri, J. and Mueller, B. (2007). Why has the share of training firms } \\
\text { declined in Switzerland. ZAF } 2 \text { und 3/2007, S. 149-167 }\end{array}$ \\
\hline 34 & $\begin{array}{l}\text { Siegenthaler, M. (2011). Can a standardised aptitude test predict the } \\
\text { training success of apprentices - Evidence from a case study in } \\
\text { Switzerland. Vocational education and training } 3 \text { (2011) 2, S. 105-128. }\end{array}$ \\
\hline 35 & $\begin{array}{l}\text { Stone Iii, J. R. and M. V. Lewis (2010). "Governance of vocational } \\
\text { education and training in the United States." Research in Comparative and } \\
\text { International Education } 5(3): 274-288 .\end{array}$ \\
\hline 36 & $\begin{array}{l}\text { Wallis. P (2007). Apprenticeship and Training in Premodern England. } \\
\text { LSE No. 22/07 }\end{array}$ \\
\hline 37 & $\begin{array}{l}\text { Wolter, S. C., et al. (2006). "Why some firms train apprentices and many } \\
\text { others do not." German Economic Review 7(3): 249-264. }\end{array}$ \\
\hline
\end{tabular}

